\title{
MODULES WITH ARTINIAN PRIME FACTORS
}

\author{
EFRAIM P. ARMENDARIZ
}

\begin{abstract}
An $R$-module $M$ has Artinian prime factors if $M / P M$ is an Artinian module for each prime ideal $P$ of $R$. For commutative rings $R$ it is shown that Noetherian modules with Artinian prime factors are Artinian. If $\boldsymbol{R}$ is either commutative or a von Neumann regular $V$-ring then the endomorphism ring of a module with Artinian prime factors is a strongly $\pi$-regular ring.
\end{abstract}

A ring $R$ with 1 is left $\pi$-regular if for each $a \in R$ there is an integer $n \geqslant 1$ and $b \in R$ such that $a^{n}=a^{n+1} b$. Right $\pi$-regular is defined in the obvious way, however a recent result of F. Dischinger [5] asserts the equivalence of the two concepts. A ring $R$ is $\pi$-regular if for any $a \in R$ there is an integer $n \geqslant 1$ and $b \in R$ such that $a^{n}=a^{n} b a^{n}$. Any left $\pi$-regular ring is $\pi$-regular but not conversely. Because of this, we say that $R$ is strongly $\pi$-regular if it is left (or right) $\pi$-regular.

In [2, Theorem 2.5] it was established that if $R$ is a (von Neumann) regular ring whose primitive factor rings are Artinian and if $M$ is a finitely generated $R$-module then the endomorphism ring $\operatorname{End}_{R}(M)$ of $M$ is a strongly $\pi$-regular ring. Curiously enough, the same is not true for finitely generated modules over strongly $\pi$-regular rings, as Example 3.1 of [2] shows. Obviously, it also fails for arbitrary regular rings. These observations lead one to consider conditions on finitely generated modules which ensure that the endomorphism ring is strongly $\pi$-regular. A natural one seems to be that of having Artinian prime factors. In fact, we establish that such modules have strongly $\pi$-regular endomorphism ring whenever the base ring is either commutative or a regular $V$-ring.

Consider a finitely generated module $M$ over a ring $R$. If $R$ is commutative then $R$ is $\pi$-regular if and only if its prime ideals are maximal [11]. Accordingly, when $R$ is commutative and $\pi$-regular, $M / P M$ is an Artinian module for all primes $P$. This observation serves as a starting point.

THEOREM 1. Suppose $R$ is a commutative ring. For a finitely generated $R$-module $M$ the following conditions are equivalent.

(a) $M$ has Artinian prime factors.

(b) $S=\operatorname{End}_{R}(M)$ is a strongly $\pi$-regular ring.

(c) $R / \operatorname{Ann}_{R}(M)$ is a $\pi$-regular ring.

Received by the editors September 19, 1978 and, in revised form, April 17, 1979.

AMS (MOS) subject classifications (1970). Primary 16A30, 16A46, 16A64.

Key words and phrases. Artinian module, endomorphism ring, strongly $\pi$-regular ring, von Neumann regular ring. 
Proof. (b) $\Rightarrow$ (c): Since $M$ is finitely generated, $S$ satisfies a polynomial identity. Hence each prime ideal of $S$ is a maximal ideal (see e.g. [1]). Now $R^{\prime}=$ $R / \operatorname{Ann}_{R}(M)$ embeds in the center of $S$ and $S$ is an integral extension of $R^{\prime}$ [10]. It follows that prime ideals of $R^{\prime}$ are maximal ideals [3] and hence $R^{\prime}$ is $\pi$-regular.

(c) $\Rightarrow(a)$ : This is clear.

(a) $\Rightarrow$ (b): By [2, Proposition 2.3], $S$ is strongly $\pi$-regular if and only if for each $\alpha \in S$ there is an integer $t \geqslant 1$ such that $M=\operatorname{Ker} \alpha^{t} \boxplus M \alpha^{t}$. Thus let $\alpha \in S$. Our first step is to show that there is an integer $t \geqslant 1$ such that $M \alpha^{t}=M \alpha^{t+1}$. Assume that no such integer exists. Because $M$ is finitely generated, there is an ideal $P$ of $R$ which is maximal among those ideals $I$ of $R$ having the property that $M \alpha^{k} \varepsilon$ $M \alpha^{k+1}+I M$ for all integers $k \geqslant 1$. We claim that $P$ is a prime ideal of $R$. Thus suppose $A$ and $B$ are ideals of $R$ properly containing $P$ and such that $A B \subseteq P$. We then have integers $m, n$ such that $M \alpha^{m} \subseteq M \alpha^{m+1}+A M$ and $M \alpha^{n} \subseteq M \alpha^{n+1}+$ $B M$. The second of these inclusions gives us $A M \alpha^{n} \subseteq A M \alpha^{n+1}+P M$, since $A B \subseteq P$. Hence $A M \alpha^{n+1} \subseteq A M \alpha^{n+2}+P M$, giving $A M \alpha^{n} \subseteq A M \alpha^{n+2}+P M$. Continuing we arrive at $A M \alpha^{n} \subseteq A M \alpha^{n+m+1}+P M$ and therefore $A M \alpha^{n} \subseteq$ $M \alpha^{n+m+1}+P M$. Using this we then get $M \alpha^{n+m}=\left(M \alpha^{m}\right) \alpha^{n} \subseteq\left(M \alpha^{m+1}+\right.$ $A M) \alpha^{n} \subseteq M \alpha^{n+m+1}+P M$, which contradicts the choice of $P$. Thus $P$ is a prime ideal as claimed. By assumption, $M / P M$ is an Artinian module. But then the sequence of submodules $M \alpha \supseteq M \alpha^{2} \supseteq \cdots$ must terminate modulo $P M$, providing the desired contradiction. This shows then that $M \alpha^{t}=M \alpha^{t+1}$ for some integer $t \geqslant 1$. Now $\alpha$ is an onto endomorphism of the finitely generated $R$-module $M \alpha^{t}$, and so $\alpha$ is $1-1$ on $M \alpha^{t}$ since $R$ is commutative [12]. Then $\operatorname{Ker} \alpha \cap M \alpha^{t}=0$ implies that $\operatorname{Ker} \alpha^{t}=\operatorname{Ker} \alpha^{t+1}$. It now follows easily that $M=\operatorname{Ker} \alpha^{t} \oplus M \alpha^{t}$, completing the proof.

Examination of the proof of the implication $(a) \Rightarrow(b)$ shows that commutativity was used only to ensure that onto endomorphisms are 1-1. It has been shown in [2, Theorem 2.2] that rings integral over their center and satisfying a polynomial identity have the property that onto endomorphisms of finitely generated modules are 1-1, a property which left Noetherian rings also have. Thus we are able to state the following.

Theorem 2. Assume $R$ is either a PI-ring integral over its center or a left Noetherian ring. If $M$ is a finitely generated left $R$-module having Artinian prime factor then $\operatorname{End}_{R}(M)$ is a strongly $\pi$-regular ring.

In view of this theorem one might ask if any Noetherian $R$-module with Artinian prime factors is Artinian. The answer is no, in general. An example in [9, p. 66] provides us with a perfect ring $D$ having a Noetherian non-Artinian module. Since $D / P$ is simple Artinian for any prime ideal $P$, such a module must have Artinian prime factors. Before showing that the answer is affirmative when $R$ is commutative, we note that over a semiprimary ring, all Noetherian modules are Artinian, so the answer is (trivially) yes in this case.

THeOREM 3. If $R$ is a commutative ring and $M$ is a Noetherian $R$-module with Artinian prime factors then $M$ is Artinian. 
Proof. By Theorem $1, R^{\prime}=R / \operatorname{Ann}_{R}(M)$ is a $\pi$-regular ring. Since $M$ is a faithful finitely generated $R^{\prime}$-module, $R^{\prime}$ is isomorphic to a submodule of a finite direct sum of copies of $M$. Hence $R^{\prime}$ is a Noetherian module. Any Noetherian $\pi$-regular ring is Artinian so $R^{\prime}$ is Artinian. But then $M$, being a finitely generated $R^{\prime}$-module, must be Artinian.

While it is false in general that Noetherian modules with Artinian prime factors are Artinian, the following is true.

TheOREM 4. If $M$ is a Noetherian $R$-module with Artinian prime factors then $S=\operatorname{End}_{R}(M)$ is semiprimary.

Proof. The proof of (a) $\Rightarrow(\mathrm{b})$ shows that $S$ is a strongly $\pi$-regular ring. Thus each nonnil one sided ideal contains a nonzero idempotent. It follows that $J(S)$, the Jacobson radical of $S$, is a nil ideal. By a theorem of L. Small (see [6, Theorem 2.1]), nil subrings of $S$ are nilpotent, so that $J(S)$ is a nilpotent ideal of $S$. Now orthogonal idempotents of $S / J(S)$ can be lifted to $S$. However $M$ is Noetherian so $S$ can have no infinite set of orthogonal idempotents. It follows then that $S / J(S)$ is a semisimple Artinian ring.

This theorem generalizes the well-known fact that the ndomorphism ring of a Noetherian Artinian module is semiprimary.

We now turn to a result which covers [2, Theorem 2.3]. Recall that a (left) $V$-ring is a ring all of whose simple left modules are injective. For the salient features of $V$-rings we refer the reader to [4, Chapter 5].

TheOREM 5. Assume $R$ is a regular $V$-ring. If $M$ is a finitely generated $R$-module with Artinian prime factors then $S=\operatorname{End}_{R}(M)$ is a strongly $\pi$-regular ring.

Proof. Let $\alpha \in S$. As in the proof of Theorem 1, there is an integer $t \geqslant 1$ such that $M \alpha^{t}=M \alpha^{t+1}$. Suppose $x \in \operatorname{Ker} \alpha^{t+1}$; if $u=x \alpha^{t} \neq 0$, then there is an ideal $P$ of $R$ maximal among those ideals $I$ of $R$ for which $u \notin I M$. Hence $u \in A M$ for all ideals $A$ of $R$ properly containing $P$. If $P$ is not a prime ideal then there are ideals $A$ and $B$ of $R$ properly containing $P$ for which $A B \subseteq P$. Then $u \in B M$ so that $A u \subseteq A B M \subseteq P M$. Since we also have $u \in A M$ we can write $u=\Sigma a_{i} m_{i}$ where $a_{i} \in A, m_{i} \in M$. Because $R$ is a regular ring there is an idempotent $e \in A$ such that $e a_{i}=a_{i}$ for each $i$. But then $u=e u \in A u \subseteq P M$, a contradiction. Thus $P$ must be a prime ideal and the module $M / P M$ is Artinian. Because $R$ is a $V$-ring and $M / P M$ has finitely generated essential socle, we infer that $M / P M$ is completely reducible and hence Noetherian. Then $\alpha$ induces $\beta \in \operatorname{End}_{R}(M / P M)$ and $(M / P M) \beta^{t}=(M / P M) \beta^{t+1}$ and this yields $\operatorname{Ker} \beta^{t}=\operatorname{Ker} \beta^{t+1}$. But then $x \alpha^{t} \in$ $P M$, which is the desired contradiction. It now follows that $\operatorname{Ker} \alpha^{t}=\operatorname{Ker} \alpha^{t+1}, M$ $=M \alpha^{t} \oplus \operatorname{Ker} \alpha^{t}$, and so $S$ is strongly $\pi$-regular.

COROllaRy 5 [2, TheOREM 2.3]. If $R$ is a regular ring whose primitive factor rings are Artinian then $\operatorname{End}_{R}(M)$ is strongly $\pi$-regular for any finitely generated $R$-module $M$. 
Proof. It is enough to note that (i) $R$ is a $V$-ring, and (ii) prime factor rings of $R$ are Artinian. That (i) holds follows from [4, Corollary 5.13] while [8, Theorem 3, p. 239] guarantees (ii).

It is straightforward to see that a finitely generated projective Artinian module over a semiprime ring is completely reducible. Thus the proof of Theorem 4 can be used to prove the next result.

THEOREM 6. Let $R$ be a regular ring and $M$ a finitely generated $R$-module. If $M / P M$ is a projective Artinian $R / P$-module for each prime ideal $P$ of $R$ then $\operatorname{End}_{R}(M)$ is strongly $\pi$-regular.

In the first version of this article we asked whether or not a finitely generated Artinian module over a regular ring is Noetherian. An affirmative answer would then imply the statement,

over any regular ring, finitely generated modules with Artinian prime factors have a strongly $\pi$-regular endomorphism ring.

Recently, K. Goodearl has constructed examples of cyclic Artinian nonNoetherian modules as well as Noetherian non-Artinian modules over regular rings [7]. Thus our original question has a negative answer. However the validity of (*) still remains open, and would be true should the following question have a positive response. If $M$ is a finitely generated Artinian module over a regular ring, is every onto endomorphism of $M$ also 1-1?

\section{REFERENCES}

1. E. P. Armendariz and J. W. Fisher, Regular P.I.-rings, Proc. Amer. Math. Soc. 39 (1978), 247-251.

2. E. P. Armendariz, J. W. Fisher and R. L. Snider, On injective and surjective endomorphisms of finitely generated modules, Comm. Algebra 6 (1976), 659-672.

3. W. B. Blair, Right Noetherian rings integral over their centers, J. Algebra 27 (1973), 187-198.

4. J. Cozzens and C. Faith, Simple Noetherian rings, Cambridge Tracts in Mathematics, no. 69, Cambridge Univ. Press, Cambridge, 1975.

5. F. Dischinger, On strongly $\pi$-regular rings, C. R. Acad. Sci. 285 (1976), 571-573.

6. J. W. Fisher, Nil subrings of endomorphism rings of modules, Proc. Amer. Math. Soc. 34 (1972), 75-78.

7. K. Goodearl, Artinian and Noetherian modules over regular rings (to appear).

8. N. Jacobson, Structure of rings, Amer. Math. Soc. Colloq. Publ., vol. 37, Amer. Math. Soc., Providence, R. I., 1956.

9. R. W. Miller and D. R. Turnidge, Some examples from infinite matrix rings, Proc. Amer. Math. Soc. 38 (1973), 65-67.

10. M. Orzech, Onto endomorphisms are isomorphisms, Amer. Math. Monthly 78 (1971), 357-362.

11. H.-H. Storrer, Epimorphismen von Kommutativen Ringen, Comment. Math. Helv. 43 (1968), $387-401$.

12. W. V. Vasconcelos, On finitely generated flat modules, Trans. Amer. Math. Soc. 138 (1969), 505-512.

Department of Mathematics, University of TeXas, Austin, Texas 78712 